\title{
Looking for strangeness with neutrino-nucleon scattering
}

\author{
W.M. Alberico ${ }^{1}$, S.M. Bilenky ${ }^{2}$, and C. Maieron ${ }^{3}$ \\ ${ }^{1}$ Dipartimento di Fisica Teorica, Università di Torino \\ and INFN, Sezione di Torino, Via P. Giuria 1, 10125 Torino, Italy \\ ${ }^{2}$ Joint Institute for Nuclear Research, Dubna, Russia \\ ${ }^{3}$ INFN, Sezione di Catania, Via S. Sofia 64, I-95123 Catania, Italy
}

\begin{abstract}
The possibility to determine the axial strange form factor of the nucleon from elastic neutrino-nucleon scattering experiments is studied. The existing experimental information is shortly mentioned and several observables which could be measured in the near future at new neutrino facilities are discussed.
\end{abstract}

\section{Introduction}

The measurement of the cross-sections for neutral current (NC) neutrino (antineutrino) nucleon elastic scattering

$$
\nu_{\mu}\left(\bar{\nu}_{\mu}\right)+N \longrightarrow \nu_{\mu}\left(\bar{\nu}_{\mu}\right)+N
$$

has been indicated as a key tool for the determination of the so-called strange form factor of the nucleon, namely the matrix element of the (isoscalar) strange axial current:

$$
\left\langle p^{\prime}\left|\bar{s} \gamma^{\alpha} \gamma^{5} s\right| p\right\rangle=\bar{u}\left(p^{\prime}\right) \gamma^{\alpha} \gamma^{5} u(p) G_{A}^{s}\left(Q^{2}\right)
$$

Here $s, \bar{s}$ is the strange quark field operator, $|p\rangle\left(\left|p^{\prime}\right\rangle\right)$ is the state vector of a nucleon with momentum $p\left(p^{\prime}\right)$. The $\mathrm{NC}$ which intervenes in the process (11) is:

$$
J_{\alpha}^{Z}=V_{\alpha}^{3}+A_{\alpha}^{3}-2 \sin ^{2} \theta_{W} J_{\alpha}^{e m}-\frac{1}{2} V_{\alpha}^{s}-\frac{1}{2} A_{\alpha}^{s}
$$


It contains the customary vector and axial isovector components, which are the third components of isovectors, the electromagnetic current and the strange (isoscalar) axial and vector currents (though heavier quarks could come into play as well).

In order to disentangle the tiny effect (the present estimates are of the order of a few \%) of the strange form factors, various observables have been suggested, all of them being ratios of cross sections: these quantities have the advantage of minimizing the uncertainties connected with, e.g., the determination of the $\nu$-flux and/or the influence of the nuclear medium, when $\nu(\bar{\nu})$ are scattered off nuclei $[1]$.

Let us thus consider the following quantities:

1. $\mathrm{NC}$ over CC ratio:

$$
R_{N C / C C}\left(Q^{2}\right)=\frac{\left(d \sigma / d Q^{2}\right)_{\nu}^{N C}}{\left(d \sigma / d Q^{2}\right)_{\nu}^{C C}}
$$

2. Neutrino-antineutrino asymmetry:

$$
\mathcal{A}\left(Q^{2}\right)=\frac{\left(\frac{d \sigma}{d Q^{2}}\right)_{\nu}^{N C}-\left(\frac{d \sigma}{d Q^{2}}\right)_{\bar{\nu}}^{N C}}{\left(\frac{d \sigma}{d Q^{2}}\right)_{\nu}^{C C}-\left(\frac{d \sigma}{d Q^{2}}\right)_{\bar{\nu}}^{C C}}
$$

The cross sections in the denominators refer to the charged current (CC) processes:

$$
\begin{aligned}
& \nu_{\mu}+n \longrightarrow \mu^{-}+p \\
& \bar{\nu}_{\mu}+p \longrightarrow \mu^{+}+n
\end{aligned}
$$

and can be determined with higher accuracy, since all particles in the final state can, in principle, be detected, while the final $\nu(\bar{\nu})$ in the NC process (11) is not observed.

The elastic NC $\nu(\bar{\nu})$-nucleon scattering can be written in the form:

$$
\begin{aligned}
& \left(\frac{d \sigma}{d Q^{2}}\right)_{\nu(\bar{\nu})}^{N C}=\frac{G_{F}^{2}}{2 \pi}\left[\frac{1}{2} y^{2}\left(G_{M}^{N C}\right)^{2}+\left(1-y-\frac{M}{2 E} y\right) \frac{\left(G_{E}^{N C}\right)^{2}+\frac{E}{2 M} y\left(G_{M}^{N C}\right)^{2}}{1+\frac{E}{2 M} y}+\right. \\
& \left.\quad+\left(\frac{1}{2} y^{2}+1-y+\frac{M}{2 E} y\right)\left(G_{A}^{N C}\right)^{2} \pm 2 y\left(1-\frac{1}{2} y\right) G_{M}^{N C} G_{A}^{N C}\right]
\end{aligned}
$$


In the above formula $y=p \cdot q / p \cdot k=Q^{2} / 2 p \cdot k, E$ is the $\nu(\bar{\nu})$ energy in the laboratory system, $p(k)$ the initial nucleon (neutrino) four-momentum, $M$ is the nucleon mass and $Q^{2}=-q^{2}$ the square of the four-momentum transfer. Moreover $G_{E}^{N C}, G_{M}^{N C}, G_{A}^{N C}$ are the electric, magnetic and axial weak NC form factors of the nucleon, all of them containing an isoscalar strange component. In particular

$$
G_{A}^{N C ; p(n)}\left(Q^{2}\right)= \pm \frac{1}{2} G_{A}\left(Q^{2}\right)-\frac{1}{2} G_{A}^{s}\left(Q^{2}\right),
$$

where the strange axial form factor can be parameterized with the usual dipole form $G_{A}^{s}\left(Q^{2}\right)=g_{A}^{s} /\left(1+Q^{2} / M_{A}^{2}\right)^{2}$, with $g_{A}^{s}=G_{A}^{s}(0)$.

From Equation (6) it is clear that to the NC scattering process several unknown quantities contribute: in particular there are three (electric, magnetic and axial) strange form factors, two of which $\left(G_{M}^{s}\right.$ and $\left.G_{A}^{s}\right)$ can produce contributions of similar size. Moreover it has been pointed out 2 that the present uncertainty on the axial cutoff mass, $M_{A}=1.032 \pm 0.036 \mathrm{GeV}$, allows one to obtain equally good fits to the elastic $\nu-N$ scattering cross sections with values of $\left|g_{A}^{s}\right|$ ranging from 0 to 0.25 .

\section{The $\nu-\bar{\nu}$ Asymmetry}

From the explicit evaluation of the NC and CC $\nu(\bar{\nu})$-nucleon cross sections, one can express the neutrino-antineutrino asymmetry as follows $[\underline{3}$ :

$$
\mathcal{A}_{p(n)}=\frac{1}{4}\left( \pm 1-\frac{G_{A}^{s}}{G_{A}}\right)\left( \pm 1-2 \sin ^{2} \theta_{W} \frac{G_{M}^{p(n)}}{G_{M}^{3}}-\frac{1}{2} \frac{G_{M}^{s}}{G_{M}^{3}}\right)
$$

where the $+(-)$ sign refer to proton (neutron) respectively. Taking into account only terms which linearly depend on the strange form factors:

$$
\mathcal{A}_{p(n)}=\mathcal{A}_{p(n)}^{0} \mp \frac{1}{8} \frac{G_{M}^{s}}{G_{M}^{3}} \mp \frac{G_{A}^{s}}{G_{A}} \mathcal{A}_{p(n)}^{0}
$$

we find out that any deviation with respect to the (known) term,

$$
\mathcal{A}_{p(n)}^{0}=\frac{1}{4}\left(1 \mp 2 \sin ^{2} \theta_{W} \frac{G_{M}^{p(n)}}{G_{M}^{3}}\right),
$$

must be ascribed to a non-vanishing contribution of $G_{M}^{s}$ and/or $G_{A}^{s}$. 
Measurements of the asymmetry (8) is a quite demanding task from the experimental point of view, since it requires both $\nu$ and $\bar{\nu}$ beams of comparable intensity. An indirect "experimental" value of this asymmetry with flux-averaged cross sections was extracted [4] from the data of the BNL-734 experiment [2]. We refer the reader to ref. [4] for the details of the analysis. The main conclusion, however, was that the present experimental uncertainty is compatible with any value of $g_{A}^{s}$, in the range $0 \geq g_{A}^{s} \geq-0.12$.

From Equation (9), the interference between the magnetic $\left(G_{M}^{s}\right)$ and axial $\left(G_{A}^{s}\right)$ strange form factors is evident: should they have the same sign, then their effects on the asymmetry get enhanced. The opposite is true, however, if they have opposite sign.

\section{$3 \quad$ Future perspectives}

We consider here the ratio of $\mathrm{NC}$ to $\mathrm{CC}$ elastic $\nu-p$ scattering cross sections: the information on the strange form factors one can extract from this quantity is not free from ambiguities, however it deserves to be carefully considered. It was recently proposed [5] to use the high intensity Booster neutrino beam at Fermilab, to measure $\nu$-nucleon CC quasi-elastic and $\mathrm{NC}$ elastic scattering, with neutrino energies in the $0.5 \div 1.0 \mathrm{GeV}$ range. This kinematical conditions appear to be quite interesting to analyze the ratio $R_{N C / C C}\left(Q^{2}\right)$, Equation (3). From a throughout analysis we have performed on this quantity, we can summarize the following outcomes:

1. It is sensitive to $g_{A}^{s}$, but not much affected by the cutoff mass of the axial form factors, assumed in the above quoted dipole form.

2. The interference between axial and vector strange form factors (in particular the magnetic strange one) can hinder the effect of $g_{A}^{s}$ alone. However $G_{M}^{s}$ is under investigation also with polarized electron-proton scattering experiments [6] and one can hope to have complementary information from this source.

3. The sensitivity to the flux is negligible, because it is largely eliminated in the ratio of cross sections.

4. The same argument applies to nuclear medium effects: indeed a large fraction of processes would occur on ${ }^{12} \mathrm{C}$, where nucleons are bound 


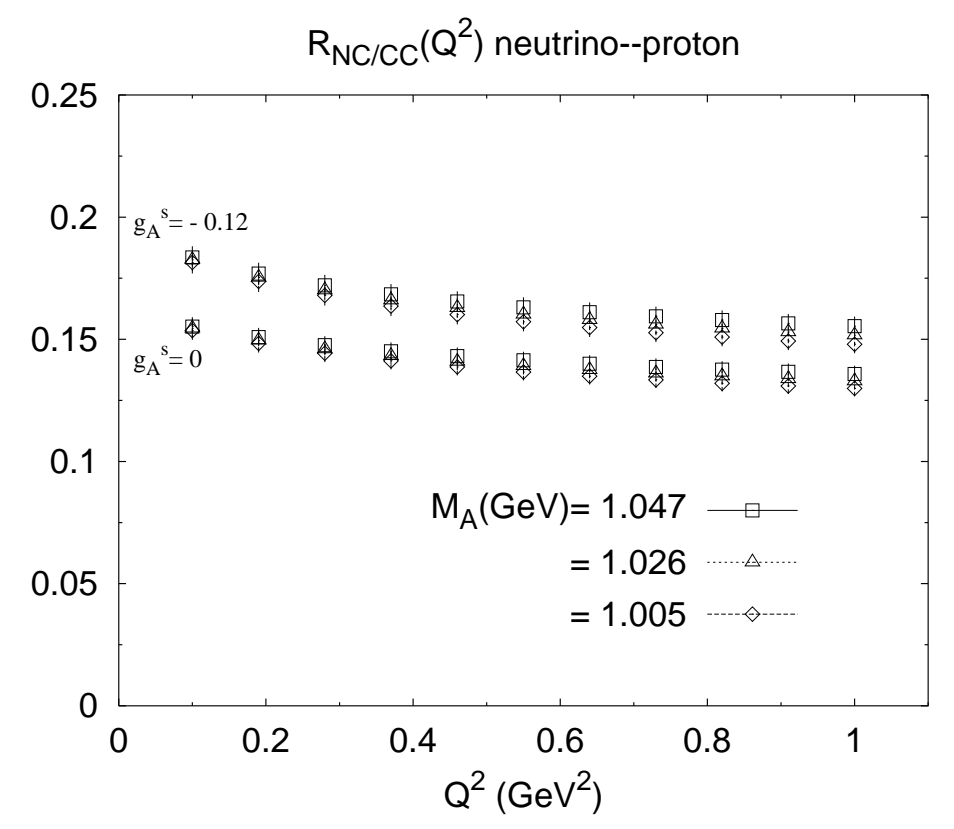

Figure 1: Plot of the ratio $R_{N C / C C}\left(Q^{2}\right)$, obtained with the neutrino cross sections averaged over the $\nu$ spectrum, for different choices of $M_{A}$ and $g_{A}^{s}$.

and subject to final state interactions. These sizeably reduce the single cross sections, but their net effect on the ratio can be safely neglected.

To illustrate some of the above points, we show in fig. 11 the $\mathrm{NC} / \mathrm{CC}$ ratio, for different choices of the axial cutoff mass $M_{A}$ and of the strange axial constant $g_{A}^{s}$, as indicated. We have assumed that this ratio could be measured with a $5 \%$ accuracy, represented by the small "error band" plotted for each calculated point. We can see that, for the moderate $Q^{2}$ values represented here, the sensitivity of this ratio to $G_{A}^{s}$ is large enough to allow a precise determination of it.

We conclude by observing that $\bar{\nu}$ scattering, if feasible, would offer relevant and complementary information on the strange form factors of the nucleon, and, eventually, would allow the determination of the neutrino asymmetry. 


\section{References}

[1] For a recent review on this subject see:

W.M. Alberico, S.M. Bilenky and C. Maieron, Phys. Rept., 358, 227 (2002).

[2] L.A. Ahrens, et al., Phys. Rev., D35, 785 (1997).

[3] W.M. Alberico, S.M. Bilenky, C. Giunti and C. Maieron, Z. Phys., C70, 463 (1996).

[4] W.M. Alberico, et al., Nucl. Phys., A651, 277 (1999).

[5] See the contribution by R. Tayloe, in these Proceedings.

[6] R. Hasty, et al. [SAMPLE Collaboration], Science, 290, 2117 (2000). 